\title{
Recovering the Star Formation History of IC 1613 Dwarf Galaxy Using Evolved Stars
}

\author{
Seyed Azim Hashemi ${ }^{1,2}$, Atefeh Javadi ${ }^{2}$ and Jacco Th. van Loon ${ }^{3}$ \\ ${ }^{1}$ Department of Physics, Sharif University of Technology, Tehran, 11155-9161, Iran \\ email: hashemi_seyedazim@physics.sharif .edu \\ ${ }^{2}$ School of Astronomy, Institute for Research in Fundamental Sciences (IPM), Tehran, \\ 19395-5531, Iran \\ email: atefeh@ipm.ir \\ ${ }^{3}$ Lennard-Jones Laboratories, Keele University, ST5 5BG, UK \\ email: j.t.van.loon@keele.ac.uk
}

\begin{abstract}
Determining the star formation history ( $\mathrm{SFH}$ ) is key to understand the formation and evolution of dwarf galaxies. Recovering the $\mathrm{SFH}$ in resolved galaxies is mostly based on deep colour-magnitude diagrams (CMDs), which trace the signatures of multiple evolutionary stages of their stellar populations. In distant and unresolved galaxies, the integrated light of the galaxy can be decomposed, albeit made difficult by an age-metallicity degeneracy. Another solution to determine the SFH of resolved galaxies is based on evolved stars; these luminous stars are the most accessible tracers of the underlying stellar populations and can trace the entire SFH. Here we present a novel method based on long period variable (LPV) evolved asymptotic giant branch (AGB) stars and red supergiants (RSGs). We applied this method to reconstruct the $\mathrm{SFH}$ for IC 1613, an irregular dwarf galaxy at a distance of $750 \mathrm{kpc}$. Our results provide an independent confirmation that no major episode of star formation occurred in IC 1613 over the past 5 Gyr.
\end{abstract}

Keywords. stars: variables: general, stars: AGB and post-AGB, stars: supergiants, galaxies: dwarf, galaxies: irregular, galaxies: individual: IC 1613

\section{Introduction}

Because of observational constraints, different methods have been developed to find the star formation history ( $\mathrm{SFH}$ ) of galaxies. Recovering the SFH in resolved galaxies is mostly based on color-magnitude diagrams (CMDs), where we can trace the signatures of most of the stellar population using high resolution observations (e.g., Tosi, Greggio \& Focardi 1989; Gallart et al. 1996; Dolphin 1997, 2002). As a matter of fact, because of the limited spatial resolution, this kind of analysis will limit us to a few dozen galaxies, mostly in our group (within $\sim 2 \mathrm{Mpc}$, Ruiz-Lara et al. 2015). In the distant and unresolved galaxies, analysis based on the individual stars is unfeasible, except possibly for the very brightest. Therefore, we are limited to the integrated light of galaxies (Ganda et al. 2009; Pérez \& Sánchez-Blázquez 2011). The integrated light of the galaxy can be decomposed, albeit made difficult by an age-metallicity degeneracy (e.g., Pickles 1985; Bica 1988; MacArthur et al. 2004). This method is relatively successful in deriving the SFHs of simple systems, but still have some limitations to give the detailed SFHs of galaxies with complex stellar compositions (Ruiz-Lara et al. 2015).

Another solution to determine the SFH of resolved galaxies is based on evolved stars (Javadi et al. 2011a,c, 2013, 2015a,b) these luminous stars are the most accessible tracers of the underlying stellar populations and can trace the entire SFH. In this paper we are going to apply a method recently developed in Javadi, van Loon \& Mirtorabi (2011b), 
in which we use long period variables (LPVs) to find the SFH. LPVs are mostly evolved asymptotic giant branch (AGB) stars (e.g., Fraser et al. 2005, 2008; Soszyński et al. 2009; Boyer et al. 2017; Goldman et al. 2019; Yuan et al. 2018), and in addition to their luminosity, their variability also helps us to identify them. This method already has been used successfully to derive SFHs of some nearby galaxies (Javadi et al. 2011b, 2016, 2017; Rezaeikh et al. 2014; Hamedani Golshan et al. 2017; Hashemi et al. 2017; Hashemi et al. 2019). In the following, we will apply this technique to find the SFH of IC 1613 dwarf galaxy.

\section{Data and Method}

IC 1613 is an isolated, irregular dwarf galaxy within the Local Group at distance of 750 kpc (Menzies et al. 2015). Its vicinity, inclination angle (Lake \& Skillman 1989), and low foreground reddening (Schlegel, Finkbeiner \& Davis 1998), makes it a great opportunity for the study of its stellar populations. Our sample to find the SFH of IC 1613 contains 53 evolved stars in the field of view of about 200 arcmin square in the central part of the galaxy. These 53 stars have been obtained using a combination of data, that were published by Menzies et al. (2015), Boyer et al. (2015). Previous application of our method (Javadi et al. 2011b, 2017; Rezaeikh et al. 2014; Hamedani Golshan et al. 2017) was based on confirmed LPVs. In the case at hand, though, the limited cadency of the DUSTiNGS survey (Boyer et al. 2015) and the limited depth of the Menzies et al. (2015) survey will have led to LPVs being missed. Therefore, we also add AGB and red supergiant (RSG) candidate stars that are expected to be LPVs to our confirmed LPVs listed in Menzies et al. (2015). These candidates are:

1-Extreme-AGB (x-AGB) stars (from Boyer et al. 2015) and large amplitude variable (LAV) stars (Menzies et al. 2015) that do not have determined period but are expected to be LPVs in the end of AGB phase (e.g., Schröder et al. 1999; Fraser et al. 2008; Soszyński et al. 2009).

2- RSG stars (Menzies et al. 2015) without determined period, but must be good candidates for being LPVs.

Using Padova evolutionary models and assuming constant metallicities, we can find mass, age and pulsation duration (duration that stars are in LPV phase) of LPVs, and then we sort the LPVs according to their ages and divide them into several bins. For different bins, with specified intervals in age and mass, we can find the star formation rate (SFR) as follows:

$$
\xi(t)=\frac{\int_{\min }^{\max } f_{\mathrm{IMF}}(m) m d m}{\int_{m(t)}^{m(t+d t)} f_{\mathrm{IMF}}(m) d m} \frac{d n^{\prime}(t)}{\delta t},
$$

where the $\mathrm{m}$ is mass, $f_{\mathrm{IMF}}(m)$ is Kroupa initial mass function (IMF) (Kroupa 2001), $d n^{\prime}$ is the observed LPVs in each bins, and $\delta t$ is pulsation duration.

The statistical error bar for each bin can be derived from Poisson distribution:

$$
\sigma_{\xi(t)}=\frac{\sqrt{N}}{N} \xi(t),
$$

where $\mathrm{N}$ is the number of stars in each age bin. 


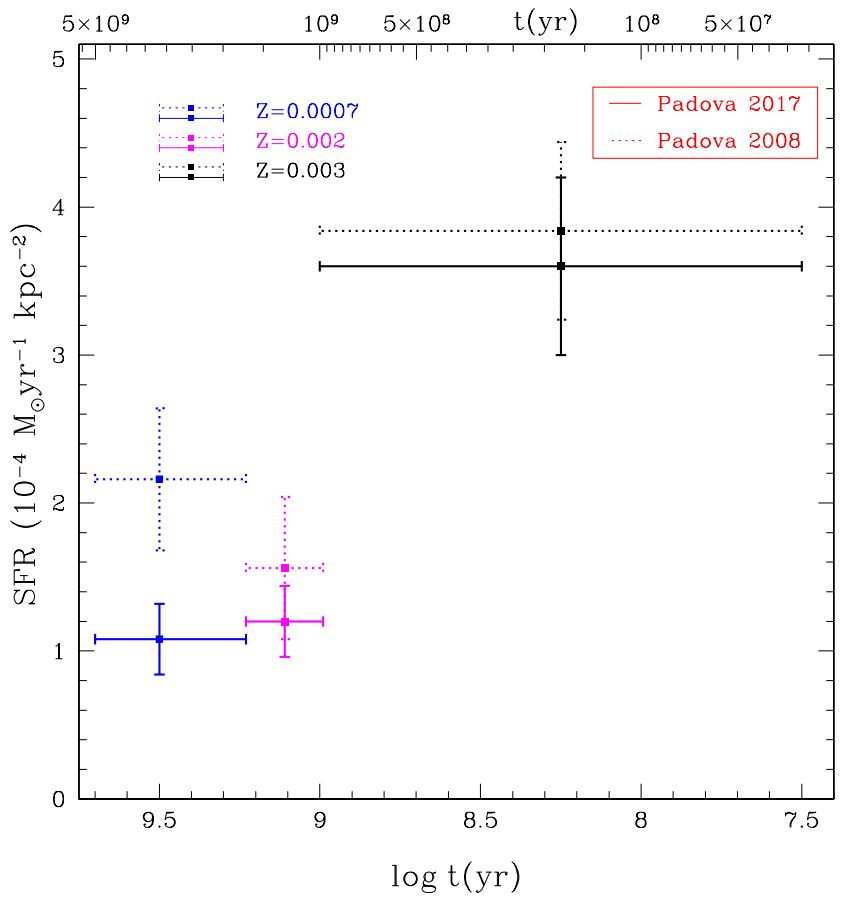

Figure 1. The SFH in IC 1613 with the adoption of age-metallicity relation that derived by Skillman et al. (2014), using the pulsation durations from Marigo et al. (2017) (solid) and Marigo et al. (2008) (dotted).

\section{Results and Discussion}

Using this new technique, we estimate SFRs in IC 1613 over the broad time interval from 30 Myr to $\sim 5$ Gyr ago. Because of the metallicity variations over time as a result of the chemical evolution due nucleosynthesis and mass loss, we adopt the linear agemetallicity relation (AMR) from Skillman et al. (2014) for values $4 \times 10^{-4}<Z<4 \times 10^{-3}$ (corresponding to 13 Gyr ago $<t<$ now). Therefore, we applied $Z=0.003$ for the last Gyr $(\log t(\mathrm{yr})<9), Z=0.002$ for 1 Gyr $<t<2$ Gyr $(9<\log t(\mathrm{yr})<9.3)$ and $Z=0.0007$ for $t>2 \mathrm{Gyr}(\log t(\mathrm{yr})>9.3)$. Considering these metallicities, our result is shown in Fig. 1. We obtained a mean value of the SFR across IC 1613 over the last Gyr of $\xi=(3.0 \pm 0.5) \times 10^{-4} \mathrm{M}_{\odot} \mathrm{yr}^{-1} \mathrm{kpc}^{-2}$, in excellent agreement with Skillman et al. (2014), who found $\xi \sim 3.4 \times 10^{-4} \mathrm{M}_{\odot} \mathrm{yr}^{-1} \mathrm{kpc}^{-2}$.

Our results, in the older time intervals $(9<\log t(\mathrm{yr})<9.6)$, are between $30 \%$ to $50 \%$ of the values derived by Skillman et al. (2014). We think that these lower rates are mainly because of incompleteness in our data (Menzies et al. 2015), however, estimation of pulsation duration $(\delta t)$ also can be a possible reason for that. In Fig.1, solid lines and symbols show the results which are obtained using 2017 Padova models (based on Marigo et al. 2017) and dotted lines and symbols show the resulting SFRs when we use the pulsation duration estimated using 2008 Padova models (based on Marigo et al. 2008). In fact, using pulsation duration from 2008 Padova models leads to higher star formation rates, which are more compatible to rates that are presented by Skillman et al. (2014). 


\section{Conclusion}

In this paper using only 53 evolved stars, we applied a novel method to find the SFH of IC 1613 dwarf galaxy. Our results show that the average star formation rate of the galaxy during the last Gyr is $\sim 3 \times 10^{-4} \mathrm{M}_{\odot} \mathrm{yr}^{-1} \mathrm{kpc}^{-2}$. Furthermore, the uniform SFH without any dominant epoch of star formation over the past 5 Gyr suggests that IC 1613 has evolved in an isolated environment at least in the past 5 Gyr.

\section{References}

Bica, E. 1988, A\&A, 195, 76

Boyer, M. L., et al. 2015, ApJ, 800, 17

Boyer, M. L., et al. 2017, ApJ, 851, 152

Dolphin, A. E., 1997, New Astron., 2, 397

Dolphin, A. E. 2002, MNRAS, 332, 91

Fraser, O. J., Hawley, S. L., Cook, K. H., Keller, S. C. 2005, AJ, 129, 768

Fraser, O. J., Hawley, S. L., Cook, K. H. 2008, AJ, 136, 1242

Ganda, K., Peletier, R. F., Balcells, M., \& Falcn-Barroso, J. 2009, MNRAS, 395, 1669

Gallart, C., Aparicio, A., Bertelli, G., Chiosi, C., 1996, AJ, 112, 1950

Hamedani Golshan, R., Javadi, A., van Loon, J. T., Khosroshahi, H., \& Saremi, E. 2017, MN$R A S, 466,1764$

Hashemi, S. A., Javadi, A. \& van Loon, J. Th. 2017, MmSAI, 88, 436

Hashemi, S. A., Javadi, A. \& van Loon, J. T. 2019, MNRAS, 483, 4751

Javadi, A., van Loon, J. T., \& Mirtorabi, M. T. 2011a, MNRAS, 411, 263

Javadi, A., van Loon, J. T., \& Mirtorabi, M. T. 2011b, MNRAS, 414, 3394

Javadi, A., van Loon, J. T., \& Mirtorabi, M. T. 2011c, ASPC, 445, 497

Javadi, A., van Loon, J. T., Khosroshahi, H., \& Mirtorabi, M. T. 2013, MNRAS, 432, 2824

Javadi, A., Saberi, M., van Loon, J. T., Khosroshahi, H., Golabatooni, N., \& Mirtorabi, M. T. 2015a, MNRAS, 447, 3973

Javadi, A., van Loon, J. T., \& Khosroshahi, H. 2015b, PKAS, 30, 355

Javadi, A., van Loon, J. T., \& Khosroshahi, H. 2016, MmSAI, 87, 278

Javadi, A., van Loon, J. T., Khosroshahi, H. G., Tabatabaei, F., Hamedani Golshan, R., \& Rashidi, M. 2017, MNRAS, 464, 2103

Kroupa, P. 2001, MNRAS, 322, 231

Lake, G., Skillman, E. D. 1989, AJ, 98, 1274

MacArthur, L. A., Courteau, S., Bell, E., \& Holtzman, J. A. 2004, ApJS, 152, 175

Marigo, P., Girardi, L., Bressan, A., Groenewegen, M. A. T., Silva, L., Granato, G. L. 2008, $A \mathscr{\xi} A, 482,883$

Marigo, P., Girardi, L., Bressan, A., Rosenfield, P., Aringer, B., Chen, Y., Trabucchi, M. 2017, ApJ, 835, 77

Menzies, J. W., Whitelock, P. A., Feast, M. W. 2015, MNRAS, 452, 910

Pickles, A. J. 1985, ApJ, 296, 340

Pérez, I. \& Sánchez-Blázquez, P. 2011, A\& A, 529, A64

Rezaeikh, S., Javadi, A., Khosroshahi, H., van Loon, J. T. 2014, MNRAS, 445, 2214

Ruiz-Lara, T., et al., 2015, A\& A, 583, A60

Saremi, E., et al. 2017, JPhCS, 869, 2068

Schlegel, D. J., Finkbeiner, D. P., Davis, M. 1998, ApJ, 500, 525

Schröder, K. P., Winters, J. M. \& Sedlmayr, E. 1999, A $\xi A, 349,898$

Skillman, E. D., et al. 2014, ApJ, 786, 44

Soszyński, I., et al. 2009, AcA, 59, 239

Tosi, M., Greggio, L., Focardi, P., 1989, Ap\&SS, 156, 295

Yuan, W., Macri, L. M., Javadi, A., Lin, Z., Huang, J. Z. 2018, AJ, 156, 112 